\title{
Dynamism: Reconceptualising Resilience in Recovery From Trauma
}

\author{
Zoe Wyatt ${ }^{1}$ \\ ${ }^{1}$ Hagar International, USA \\ Correspondence: Dr Zoe Wyatt, Hagar International, USA. E-mail: zoe@ facilitatewellbeing.com.mu
}

Received: October 19, 2021

Accepted: November 19, 2021

Online Published: November 26, 2021

doi:10.20849/ajsss.v6i4.954

URL: https://doi.org/10.20849/ajsss.v6i4.954

\begin{abstract}
Resilience research is currently dominated by Western theoretical frameworks, concepts, constructions, worldviews and understandings. Applying a Western construction of resilience to other global settings, is challenged by the fact that cultural practices central to different cultural identities, may not be acknowledged. This paper will present new and existing ways of conceptualising resilience and proposes that the term dynamism may be more culturally appropriate for capturing the concept of resilience in cross-cultural settings. The term dynamism has been crafted from research that initially sought to understand resilience and recovery among young Cambodian trauma survivors (See Wyatt, 2021). The research endeavoured to situate the concept of resilience within a non-Western, Cambodia-specific context. This study looked at factors that enabled young people to move forward with their lives and do remarkably well, despite significant childhood trauma (Wyatt, 2021). This paper is an extension of the key findings and will focus on the construction of the term resilience and its usefulness in cross-cultural settings. Furthermore, this article will present a model for dynamism and how this may be helpful in understanding recovery from trauma in cross-cultural contexts.
\end{abstract}

Keywords: cross-cultural resilience, dynamism, Cambodia, youth, trauma, recovery

\section{Introduction}

A large body of work in the resilience space is informed by American and European researchers, neuroscientists and psychologists. Early models of resilience began to emerge with the work of Werner and Smith (1982), Anthony and Cohler (1987), Masten (1989), Rutter and Team (1998) and Gottlieb (1998). These researchers' early influential work recognised the strikingly different variations in adaptive functioning of at-risk young people due to trauma, poverty, genetics and family history. As a result of this early research, there are now multiple pathways of resilience theory and science converging within an integrated resilience framework. However, despite interest in resilience theory and ongoing academic debate, the term resilience continues to be contentious.

A common definition of resilience is the ability to positively adapt to adversity (Masten, 1989). Severe adversity can be defined as an ongoing state of serious or continued difficulty, often associated with children of war and displacement studies (Krause \& Sharples, 2000). It has been argued that resilience in the face of severe adversity is a two-dimensional construct that captures both the exposure to adversity and the process of positive adjustment, also known as dynamic positive adaptation (Luthar et al., 2000; Masten, 1999). This term does not reflect a personal attribute or individual characteristic, but rather a process where the individual adapts, despite experiencing significant trauma or severe adversity (Luthar et al., 2000; Masten, 1999). Resilient individuals may demonstrate an ability to recover after serious or life-threatening events (Masten, 2015). According to von Eye and Schuster (2000), understanding resilience and a young person's ability to adapt amidst destructive environments, may enable us to determine appropriate interventions, thus circumventing the adverse outcomes of trauma. Masten (2015) argues that resilience is a common, adaptive response, which conceptualises coping strategies and competencies that are available to most people. Additionally, coping skills, or what people do to minimise stress, are resilient ways of behaving or adjusting to the demands and challenges of stress (Heffron, 2013).

Some researchers argue that the resilience remains a "simplistic view" and an "outcome of children's access to protective factors" (Skovdal \& Daniel, 2012, p.160). Western theoretical frameworks consistently maintain that resilience is possible, when exposure to considerable adverse conditions is met with positive adaptation. Children without adequate parental or familial support have been found to be at greater risk of not being able to 
cope effectively (McAdam-Crisp, 2006). Other definitions of resilience include the ability to "bounce back," or to recover from adversity by returning to pre-traumatic states of being (Smith et al., 2008; McHenry et al., 2014). Alternatively, Luthar et al. (2000) argue that "resilience refers to a dynamic process encompassing positive adaptation within the context of significant adversity" (p. 543). This approach moves away from pathologising mental illness and towards developing approaches that build thriving individuals and communities, which is a foundational starting point for real-world applications of resilience (Heffron, 2013).

Resilience, seen as positive adaptation (a component of positive psychology), is comprised of protective factors that are essential for resilience development (Dyer \& McGuinness, 1996; Heffron, 2013). Researchers Stewart and Yuen (2011) view resilience as context-dependent, where protective environmental and resilience factors overlap, mediate, or depend on each other. These factors include certain character traits, social support, age, sex, ethnicity, cognitive ability, socioeconomic status, brain function, genetics, spirituality and life history. Kalisch et al. (2014) acknowledge the well-documented influences of socio-environmental factors on mental health, which impact the development of resilience, while also focusing on building social relationships and improving the physical environment for promoting mental health and wellbeing (Wyman et al., 2000; Janicki-Deverts \& Cohen, 2011; Sweeney et al., 2018).

Critics of the medical model underscore the importance of an integrated understanding of resilience in a culturally relevant context (Ungar, 2008; Wastell \& White, 2017). Notably, some find the dominant discourse of the medical model and PTSD diagnostic criteria inaccurate for non-Western traumatised populations (Agger, 2015; Chhim, 2013; Greene, 2008). The intersectional field of psychology and social work attempts to look at more holistic interventions and Eastern philosophies that are not consistent with Western, individualistic models of personal agency and self-efficacy (Bandura, 1977; 1997). For example, the role of faith and religion in building resilience in recovery from trauma is in contrast to the medical model of resilience (Ungar, 2008), which favours a self-efficacy framework (Bandura, 1977; McHenry et al., 2014; Luthar et al., 2000; Smith et al., 2013). Additionally, faith, spirituality and supportive relationships formed through faith-based institutions, have been suggested as being resilient approaches (Masten, 2015; Miles et al., 2020). Moreover, there is a need for theoretical models to include cultural pluralism in order to increase cross-cultural validity (Mollica et al., 2014).

Promisingly, after decades of preoccupation with pathology, attitudes about trauma survivors and treatment have gradually changed, moving away from a deficit approach to a strengths-based perspective (Wastell \& White, 2017). This perspective can be particularly useful in cross-cultural settings when paired with a culturally sensitive growth-centred model of resilience (Wastell \& White, 2017; West, 2000). A culturally sensitive growth-centred model considers the development of kinship bonds and social connectedness through community in culturally meaningful ways (Wong \& Wong, 2006).

It has been argued that while health professionals working with trauma survivors use psychosocial guidelines that function effectively in the West, they often do not consider cultural context as an explanatory model that is being used by survivors to build resilience (Overland, 2013). Research with Cambodian trauma survivors tends to focus on the "non-resilient" group (Overland, 2013). Furthermore, there is limited research on how the current Western construct of resilience applies to populations outside of the West, particularly within marginalised groups, such as young people, although some studies have explored resilience in cross-cultural settings (Boyden \& Mann, 2005; Masten, 2018; Ungar, 2004).

While there is significant research into trauma and resilience, most of it is defined according to Western frameworks and there has been minimal inquiry into how resilience is defined in cross-cultural settings (Masten, 2014). This study aimed to address an important gap in the current literature regarding Cambodian young people who have experienced significant trauma. The goal was to explore the concept of resilience through the lived experiences of young Cambodians. This study incorporates elements of the above conceptualisation of resilience, taking into consideration the importance of individual resilient strategies as well as contextual and environmental factors. This research was grounded in the importance of Cambodia's sociocultural context for understanding the resilience of the young trauma survivors that participated in this study and factors facilitating resilience identified by Western researchers which can be cross-culturally applicable, suggesting commonalities shared by people overcoming trauma. This study aimed to provide insight into the construct of resilience post-trauma in a Cambodian context.

\section{Method}

This research sought to understand factors promoting the recovery and wellbeing of young Cambodian trauma survivors (Wyatt, 2021). The focus on resilience and recovery was adapted as a strengths-based framework, rather than pathologising trauma survivors. This was consistent with Antonovsky's (1987) approach of identifying those 
that were "doing remarkably well" as a means of defining resilience. The participants of this study were all Cambodian young people who had experienced traumatic events in their childhood, yet appeared to be doing remarkably well. An exploratory case study approach was used drawing upon grounded theory (GT) (Wyatt, 2021). In-depth interviews were conducted with 26 participants in the form of oral narratives, with narratives being supported by a timeline and a resilience scale (Wyatt, 2021). Field work commenced in 2019, as did many informal discussions with a network of individuals residing in Phnom Penh, as to the nature of resilience. This network included interpreters, language teachers, psychologists and social workers already known to the researcher.

The researcher spent 18 months in Cambodia and hired a Khmer tutor immediately upon arrival before commencing field work. However, Khmer is a linguistically challenging language to learn because it is very literal. As such, the abstract and psychological concepts dealt with in the research were too far advanced a language level to be obtained during the short time the researcher spent in country. For that reason, a Research Assistant (RA) was used for 12 interviews as an interpreter, except in the instance where prior communication with a young person indicated a high level of English (Wyatt, 2021). Interpreters are frequently used in health research even though this can present challenges in the interpretation meaning (Bramberg \& Sandman, 2013; Lucas, 2014). Temple and Edwards (2002) used the term "cross-language research" to describe qualitative studies that utilise an interpreter or translator. When a language barrier exists between qualitative researchers and their participants, the research becomes a cross-language qualitative study with unique challenges related to language (Edwards, 1998; Temple \& Edwards, 2002; Temple \& Young, 2004). Squires (2009) conducted a systematic review of cross-language studies in nursing and health science journals with multiple inconsistencies identified in cross-language research. Although globalisation has augmented the need for qualitative research that is linguistically and culturally representative of study participants, it is fraught with challenges (Esposito, 2001; Yach, 1992; Temple \& Edwards, 2002).

Initially the researcher began searching for words in the Khmer language to describe positive adaptation or a "resilient" equivalent. Coinciding with this, the researcher came across an article entitled "The Methodology Dynamism of Grounded Theory" (Ralph et al., 2015) in which researchers unpack the nature of Strauss and Corbin's symbolic interactionist roots, paired with the constructivism of Charmaz to describe GT's dynamism underpinnings. The authors referred to this process as "methodological dynamism characterised by contextual awareness and moment formation, contemporary translation, generational methodology" (Ralph et al., 2015, p.1). The researcher found this to be particularly relevant, as the term dynamism was beginning to have a dual meaning as a way of describing resilience. Was it possible that dynamism could in fact, be used to conceptualise a culturally relevant way of reframing resilience in Cambodia? Although dynamism emerged through the narratives of the young people and was not fully realised until much later in the analysis and writing process. The conceiving of dynamism aligned strongly with a classical GT methodology approach:

No inventor has permanent possession of the invention ... a child once launched is very much subject to the combination of its origins and the evolving contingencies of life. Can it be otherwise for a methodology? (Strauss and Corbin, 1994, p. 283).

In order to effectively translate the idea of resilience for the Cambodian context, it was necessary to find a more culturally applicable term that expresses the same idea, as resilience does not directly translate into Khmer (Quigley et al., 2011). This is because the Khmer language is very literal and as such, more abstract terminology is difficult to translate effectively as the lexicon available to English is not available in Khmer (Quigley et al., 2011). In Khmer, resilience only refers to physical objects being durable and its modern-day psychological meaning does not translate. This proved to be a challenging task of translating the word "resilience" itself into Khmer. Although this study is specifically on resilience, the researcher did not use the word anywhere in the interview questions for the Cambodian young people. Contrastingly, as many key informants were educated inside the construct of Western resilience and trauma discourses, the researcher was able to use the term in their interviews. However, the need for translatable terminology in cross-cultural sample groups (such as the participants) can be easily ameliorated by the use of alternative vocabulary that is more culturally-applicable.

\section{Result}

Care must be taken in proposing new ways that encourage a shift in focus away from the Western discourse. Furthermore, it should address the metaphorical ambiguity of the term resilience and move towards a more culturally and linguistically appropriate way of framing an outcome of agency and social interactions, within a cultural framework. Ideally, the term is accessible for both the individual and the community that wholly encompasses positive adaptation in the face of adversity. Here, dynamism is being proposed as a term which is accessible to individuals as well as cross-cultural communities, who are at the heart of this study. The term 
dynamism has the potential for us to shift away from the Westernised metaphorical ambiguity of the term resilience and towards a more culturally and linguistically appropriate way of framing the outcomes.

Furthermore, dynamism is being suggested an alternative term for resilience in Cambodia, as its Khmer equivalent sakatanopol is used abstractly and refers to potential and untapped greatness. It is culturally relevant in modern-day Cambodia, as many Cambodian marketing and media campaigns already employ the term, so everyday Cambodians are familiar with the word and its abstract meaning. Furthermore, this key word is used in many ways to describe the power and resilience of an individual and is also used in social work case management in Cambodia to indicate strength and progress (Eng, 2020). By extension, while new and unfamiliar in its usage, the conceiving of sakatanopol as dynamism in terms of the ability to positively adapt, could be suggested as culturally appropriate. Moreover, the Khmer translation of dynamism as "potential" correlates with the findings of this study as many of the survivors still have trauma symptoms but are successful in other areas of their lives. This is in direct contrast to some resilience discourses (Smith et al., 2008), which would view some study participants as not being resilient due to the presence of maladaptive coping skills, such as binge drinking and difficulties with anger management, which would be negatively framed as the individual not being recovered.

The word dynamism in English is a noun and is defined as: "1) the quality of being characterised by vigorous activity and progress; and 2) [in describing history and philosophy] the theory that phenomena of matter or mind are due to the action of forces rather than to motion or matter" (Hobson, 2004, p. 143). Extrapolating from the second definition, resilience and an individual's journey of recovery may be inferred as being "phenomena of the mind" and "the action of forces" could be described as resilience factors discussed in the data by the research participants. Dynamism as proposed is a cross-culturally relevant term for understanding the process of recovery, which is due to the action of forces, including personal attributes, external community factors, and opportunities for economic empowerment, faith and spirituality.

\section{Discussion}

In the context of modern-day Cambodia, three overarching aspects that inform dynamism are now proposed. Firstly, there are the survivor's internal forces, such as regulating their emotions and self-efficacy. These internal forces are all processes that inform Western resilience discourses and are individualistic in nature and should not be discounted. Secondly, there is the survivor's sphere of influence, which includes connection, belonging and opportunities. As mentioned previously, social connectedness has been long established as key to building resilience and sustainable recovery. Moreover, sense of belonging is a result of meaningful connection for trauma survivors. The sphere of influence also includes opportunities. The data indicated that a trauma survivor receiving opportunities to advance economically helped them establish a sense of purpose. Thirdly, the external forces present in the survivor's environment includes culture, faith and spirituality.

The findings suggest that resilience, wellbeing and recovery from trauma in Cambodia may be captured by the term sakatanopol, or dynamism. Dynamism may be understood as an internal process involving ongoing complex interactions between the individual and the external environment, bound by the cultural context, to achieve recovery from trauma Dynamism is proposed as an attempt to address the linguistic gap in translating resilience for the Cambodian context. All of this speaks to dynamism as a multiform process and journey of recovery lasting a lifetime and not something to be achieved in totality, which is sometimes inferred by resilience (Smith et al., 2008).

These external forces may vary from context to context and between cultures, however, the role of culture is important. It is the individual's culture that informs their external environment, while also shaping their internal reality and how they perceive and understand the world around them and how they create meaning (Overland, 2013; West, 2000). In the Cambodian context, the culture of the participants in this study is collectivist, which informs many sociocultural perceptions, attitudes and practices, including stigmas. Another key external force was the role of faith and spirituality in the participants' recovery, which may also be applicable in other cultural settings.

The usefulness of dynamism, in the Cambodian context as well as in other cross-cultural settings, is threefold: 1) it is a term that encompasses resilience factors captured in this study; 2) it is an emerging theory to explain recovery from trauma in more collectivist cultural settings; and 3) it is also a model as it takes into consideration sociocultural contextual factors that underpin how individuals create and understand their reality and make meaning. Dynamism as proposed may be seen in the figure below. 


\begin{tabular}{|l|l|}
\hline \multicolumn{1}{|c|}{ Resilience } & \multicolumn{1}{c|}{ Dynamism } \\
\hline $\begin{array}{l}\text { Positive adaptation to a new functional state, in } \\
\text { the context of significant adversity }\end{array}$ & $\begin{array}{l}\text { An internal process involving a set of complex } \\
\text { interactions with the external environment }\end{array}$ \\
\hline
\end{tabular}

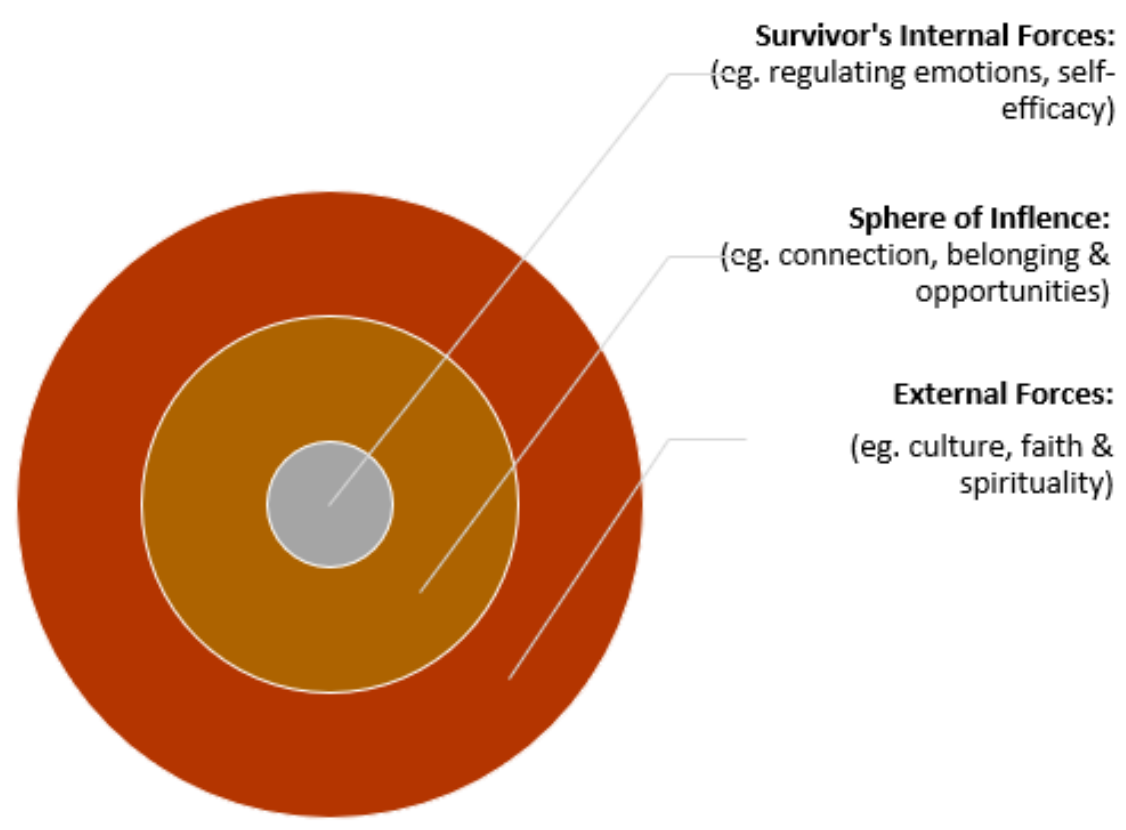

Figure 1. Proposing dynamism in cross-cultural contexts

\section{Conclusion}

This study identified the need to create a system with conditions in place that support the positive growth of trauma survivors in a cross-cultural setting, as well as a term that is more culturally relevant. Dynamism has been proposed as a strengths-based model of recovery from trauma, which emphasises growth over pathology. Importantly, this study viewed resilience from a lens of ongoing development and growth potential, concluding that dynamism may be useful and more applicable for understanding trauma in cross-cultural contexts. Future approaches to recovery from trauma could also consider if key concepts like resilience are culturally transferable and if they effectively translate into the native language of the area being studied. As resilience and other Western-centred metrics may not translate to non-Western cross-cultural contexts, in these cases, future research and work in trauma recovery could use the concept of dynamism instead.

\section{Ethics and Acknowledgements}

This article stems from $\mathrm{PhD}$ research that obtained ethics approval from both Deakin University Human Research Ethics Committee and the Cambodian Ministry of Health. The researcher is a member of the Australian Association of Social Workers was assisted by her Deakin University academic supervisors, Associate Professor Petra Staiger and Associate Professor Elizabeth Hoban.

\section{References}

Agger, I. (2015). Calming the mind: Healing after mass atrocity in Cambodia. Transcultural Psychiatry, 52(4), 543-560.

Anthony, E., \& Cohler, B. (1987). The invulnerable child. Guildford Press.

Antonovsky, A. (1987). Unraveling the mystery of health: How people manage stress and stay well. Jossey-Bass. 
Bandura, A. (1977). Self-efficacy: Toward a unifying theory of behavioral challenge. Psychological Review, 84, 191-215.

Bandura, A. (1997). Self-efficacy: The exercise of control. Freeman.

Boyden, J., \& Mann, G. (2005). Children's risk, resilience, and coping in extreme situations. In M. Ungar (Ed.), Handbook for working with children and youth: Pathways to resilience across cultures and contexts (pp. 3-26). SAGE Publications.

Bramberg, E. B., \& Sandman, L. (2013). Communication through in-person interpreters: A qualitative study of home providers and social workers' views. Journal of Clinical Nursing, 22(1-2), 159-167.

Eng, H. (2020, November 25). Interview with research assistant at Save the Children.

Esposito, N. (2001). From meaning to meaning: The influence of translation techniques on non-English focus group research. Qualitative Health Research, 11(4), 568-579.

Gottlieb, G. (1998). The significance of biology for human development: A developmental psychobiological systems view. In W. Damon, \& R. Lerner (Eds.), Handbook of Child Psychology (5th ed., Vol. 1, pp. 233-273). John Wiley \& Sons.

Greene, R. (2015). Resilience and healing among Cambodian survivors of the Khmer Rouge regime. Journal of Evidence-Informed Social Work, 12(6), 579-87.

Heffron, K. (2013). Positive psychology and the body: The somatopsychic side to flourishing. Open University Press.

Hobson, A. (2004). Oxford dictionary of difficult words. Oxford University Press.

Krause, K., \& Sharples, E. (2020). Thriving in the face of severe adversity: Understanding and fostering resilience in children affected by war and displacement. In E. Fiddian-Qasmiyeh (Ed.), Refuge in a Moving World: Tracing refugee and migrant journeys across disciplines (pp. 306-322). London: UCL Press. https://doi.org/10.2307/j.ctv13xprtw.28

Lucas, S. (2014). Social work in a multilingual world: Interpreter-mediated encounters [Doctoral dissertation, University of Stanford]. Economics and Social Work Research Council. Retrieved from http://usir.salford.ac.uk/33004/

Luthar, S. S. (2000). The construct of resilience: Applications in interventions; Keynote address, XX-XII Banff International Conference on Behavioral Sciences; Banff, AB, Canada.

Luthar, S. S., Cicchetti, D., \& Becker, B. (2000). The construct of resilience: A critical evaluation and guidelines for future work. Child Development, 71(3), 543-562.

Masten, A. (1989). Resilience in development: Implications of the study of successful adaptation for developmental psychopathology. In D. Cicchetti (Ed.), The emergence of a discipline: Rochester symposium on developmental psychopathology (Vol. 1, pp. 261-294). Lawrence Erlbaum Associates.

Masten, A. (1999). Commentary: The promise and perils of resilience research as a guide to preventive interventions. In M. D., Glantz, \& J. L. Johnson (Eds.), Resilience and development: Positive life adaptations (pp. 251-257). New York: Plenum Press.

Masten, A. (2015). Ordinary Magic: Resilience in development. The Guilford Press, New York, NY.

McAdam-Crisp, J. L. (2006). Factors that can enhance and limit resilience for children of war. Childhood, 13(4), 459-477.

McHenry, B., Sikorski, A. M., \& McHenry, J. (2014). A counselor's introduction to neuroscience. Routledge.

Miles, S., Heang, S., Lim, V., Nhanh, C., \& Sreang, P. (2020). The Butterfly Longitudinal Research Project: End of year progress report 2020. Chab Dai Coalition.

Mollica, R. F., Brooks, R., Tor, S., Lopes-Cardozo, B., \& Silove, D. (2014). The enduring mental health impact of mass violence: A community comparison study of Cambodian civilians living in Cambodia and Thailand. International Journal of Social Psychiatry, 60, 6-20. https://doi. org/10.1177/0020764012471597

National Scientific Council on the Developing Child. (2015). Supportive relationships and active skill-building strengthen the foundations of resilience (Working Paper No. 13). Center on the Developing Child, Harvard University. Retrieved from www.developingchild.harvard.edu 
Overland, G. (2013). Post traumatic survival: The lessons of Cambodian resilience. Cambrdige Scholars Publishing.

Quigley, C., Oliviera, A., Curry, A., \& Buck, G. (2011). Issues and techniques in translating scientific terms from English to Khmer for a university-level text in Cambodia. Language, Culture and Curriculum, 24(2), 159-177. https://doi.org/10.1080/07908318.2011.583663

Ralph, N., Birks, M., \& Chapman, Y. (2015) The Methodological Dynamism of Grounded Theory. International Journal of Qualitative Methods, 1-6. https://doi.org/10.1177/1609406915611576

Rogoff, B. (1990). Apprenticeship in thinking. Oxford, UK: Oxford University Press.

Rutter, M. (1998). Developmental catch-up, and deficit, following adoption after severe global early privation. Journal of Child Psychology and Psychiatry, 39, 465-476.

Seligman, M. (1975). Helplessness: On depression, development, and death. W. H. Freeman; Times Books; Henry Holt \& Co.

Skovdal, M., \& Daniel, M. (2012). Resilience through participation and coping-enabling social environments: the case of HIV-affected children in sub-Saharan Africa. African Journal of AIDS Research, 11(3), 153-164.

Smith, B., Dalen, J., Wiggins, K., Tooley, E., Christopher, P., \& Bernard, J. (2008). The brief resilience scale: Assessing the ability to bounce back. International Journal of Behavioral Medicine, 15, 194-200.

Squires, A. (2009). Methodological challenges in cross-language qualitative research: A research review. Journal of Nursing Studies, 46(2), 277-287.

Temple, B., \& Edwards, R. (2002). Interpreters/translators and cross-language research: Reflexivity and border crossings. International Journal of Qualitative Methods, 1(2), 1-12.

Temple, B., \& Young, A. (2004). Qualitative research and translation dilemmas. Qualitative Research, 4(2), 161-178.

Ungar, M. (2004). A constructionist discourse on resilience: multiple contexts, multiple realities among at-risk children and youth. Youth and Society, 35(3), 341-365.

Ungar, M. (2008). Resilience across cultures. The British Journal of Social Work, 38(2), 218-235.

Von Eye, A., \& Schuster, C. (2000). The odds of resilience. Child Development, 71(3), 563-566.

Wastell, D., \& White, S. (2017). Blinded by science: The social implications of epigenetics and neuroscience. Policy Press.

Werner, E. E., \& Smith, R. S. (1982) Vulnerable but invincible: A study of resilient children. McGraw Hill.

West, C. (2000). Pathways of thriving and resilience: Growth responses to adversity and trauma in two Cambodian communities: A comparative study between Lowell, Massachusetts and Phnom Penh, Cambodia [Doctoral dissertation]. University of Massachusetts, Lowell.

Wong P. T., \& Wong L. C. J. (2006). Handbook of multicultural perspectives on stress and coping. Spring Publications.

Wyatt, Z. (2021). Pathways of Resilience and Recovery from Trauma for Cambodian Young People. Advances in Social Science and Culture, 3(3), 80-98.

Yach, D. (1992). The use and value of qualitative methods in health research in developing countries. Social Science \& Medicine, 35(4), 603-612.

\section{Copyrights}

Copyright for this article is retained by the author(s), with first publication rights granted to the journal.

This is an open-access article distributed under the terms and conditions of the Creative Commons Attribution license (http://creativecommons.org/licenses/by/4.0/). 